\title{
A quantum electrodynamical treatment of second harmonic generation through phase conjugate six-wave mixing: Polarization analysis
}

\author{
Ian D. Hands, Shujie Lin, Stephen R. Meech, and David L. Andrews \\ School of Chemical Sciences, University of East Anglia, Norwich NR4 7TJ, United Kingdom
}

(Received 13 April 1998; accepted 17 September 1998)

\begin{abstract}
The theory underlying a six-wave mixing experiment is developed using the methods of molecular quantum electrodynamics. This general theory allows the intensity of the second harmonic radiation generated by the six-wave process to be found for arbitrary arrangements of the generating laser beams. Several different polarization geometries are treated in detail, and comparison is made to experiments performed using near-resonant conditions. The agreement is good in all cases and allows detailed information pertaining to the six-wave tensor to be extracted. The information thus obtained provides evidence of a marked departure from Kleinman symmetry. (C) 1998 American Institute of Physics. [S0021-9606(98)01948-5]
\end{abstract}

\section{INTRODUCTION}

It has been shown in a number of recent publications that high order nonlinear optical experiments are capable of providing novel information that is not available from lower order experiments. ${ }^{1-9}$ A well-known example is the nonresonant fifth-order Raman experiments, which provide detailed insights into the ultrafast dynamics of liquids. ${ }^{1-3}$ Recently, this group ${ }^{10}$ and another ${ }^{11}$ described a resonant, nondegenerate, six-wave mixing experiment. This particular experiment is of interest for two reasons. First, it leads to the generation of second harmonic radiation from a medium which is macroscopically isotropic. ${ }^{10-17}$ Second, it has been demonstrated that by employing this experiment in a time resolved geometry it is possible to extract quite new information on the orientational dynamics of solutions. ${ }^{10}$ The purpose of this paper is to treat the theory underlying a nondegenerate sixwave mixing processes in a rigorous and fully quantized manner, to compare the results with experimental measurements, and to act as a precursor to a forthcoming one on dynamical aspects. ${ }^{18}$ The theories that we propose offer an alternative to the more usual treatments based on the use of response functions. ${ }^{8,919-23}$ Given that six-wave mixing experiments are becoming increasingly common, it is important that such detailed theoretical models are developed to facilitate complete extraction of information. Here we provide a detailed quantum electrodynamical analysis of a sixwave mixing (SWM) process: The following section comprises a general theory of the SWM process and, in Sec. III the polarization dependence of the signal is considered. In Sec. IV details of the experiment are described, and in Sec. V a comparison between the experimentally measured and theoretically predicted polarization dependence is made. Good agreement between theory and experiment is obtained.

\section{THEORY}

We first develop theory for the adopted experimental arrangement shown in Fig. 1. A discussion of the optical components is deferred to Sec. IV-the important feature for the development of the theory centers on the three beams impinging on the sample, S. Of these, two are counterpropagating fundamental beams of frequency $\omega$, the third being a seeding second-harmonic beam making a small angle $\delta \sim 4.5^{\circ}$ to the fundamentals. We describe these beams as being of modes $\mathbf{m}_{1}=(\mathbf{k}, \lambda), \quad \mathbf{m}_{2}=\left(-\mathbf{k}, \lambda^{\prime}\right)$, and $\mathbf{m}_{3}$ $=\left(\mathbf{k}^{\prime}, \lambda^{\prime \prime}\right)$, where $\mathbf{k}$ is a wave vector and $\lambda$ a polarization label. The phase-conjugated second-harmonic beam is produced in the opposite direction to the seeding beam and is represented by mode $\mathbf{m}_{4}=\left(-\mathbf{k}^{\prime}, \lambda^{\prime \prime \prime}\right)$. The pulsed sources used for the experiment are carefully adjusted to ensure that all pulses arrive at the sample at the same moment in time.

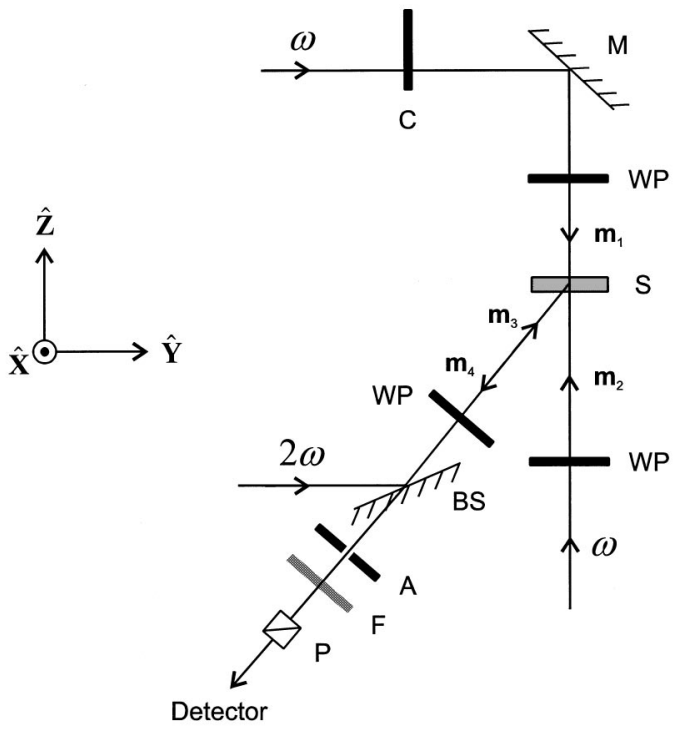

FIG. 1. Schematic diagram of the six-wave mixing experiment described in this work. The counter-propagating fundamental beams of mode $\mathbf{m}_{1}$ $=(\mathbf{k}, \lambda)$ and $\mathbf{m}_{2}=\left(-\mathbf{k}, \lambda^{\prime}\right)$, each contribute two photons while the stimulating beam, $\mathbf{m}_{3}=\left(\mathbf{k}^{\prime}, \lambda^{\prime \prime}\right)$, and signal beam, $\mathbf{m}_{4}=\left(-\mathbf{k}^{\prime}, \lambda^{\prime \prime \prime}\right)$, each gain one photon. [In the mode description each $\mathbf{k}$ refers to the wave vector and $\lambda$ to the polarization state.] Laboratory axes are as shown and other symbols represent: A-Aperture, BS-beam splitter (50:50 at $400 \mathrm{~nm}$ ), C-chopper, $\mathrm{F}-400 \mathrm{~nm}$ bandpass filter, $\mathrm{P}$ - polarizer, $\mathrm{S}$ - sample, and WP-wave plate (quarter- or half-wave). 
We shall consider an ensemble consisting of a large number of molecules all deemed to be initially in their electronic ground state. A representative Feynman diagram for the sixwave process of interest is given by Fig. 2, where we note that each fundamental mode contributes two photons to the process, the seeding (or stimulating) harmonic and signal modes each gaining one photon. The initial and final wave functions for the system will be written as

$$
\left.\begin{array}{l}
\left|\psi_{i}\right\rangle=|0\rangle\left|n\left(\mathbf{m}_{1}\right)\right\rangle\left|m\left(\mathbf{m}_{2}\right)\right\rangle\left|p\left(\mathbf{m}_{3}\right)\right\rangle\left|0\left(\mathbf{m}_{4}\right)\right\rangle \\
\left|\psi_{f}\right\rangle=|0\rangle\left|(n-2)\left(\mathbf{m}_{1}\right)\right\rangle\left|(m-2)\left(\mathbf{m}_{2}\right)\right\rangle\left|(p+1)\left(\mathbf{m}_{3}\right)\right\rangle\left|1\left(\mathbf{m}_{4}\right)\right\rangle
\end{array}\right\}
$$

where the first ket on the right refers to the molecular state and those thereafter the radiation states. In Eq. (1) the radiation is described in terms of Fock states with input modes $\mathbf{m}_{1} \cdots \mathbf{m}_{3}$ initially occupied by $n, m$, and $p$ photons, respectively, and the signal mode is empty. The equivalence for two pairs of photons implies that there are $180(=6 ! / 2 ! 2 !)$ Feynman diagrams to be considered in all; the total matrix element for molecule $\xi$ will hence be of the form

$$
M_{f i}^{(\xi)}=\chi_{(\alpha \beta)(\gamma \delta) \epsilon \phi}^{(\xi)}\left(\frac{\hbar c k}{2 \epsilon_{0} V}\right)^{2}\left(\frac{\hbar c k^{\prime}}{2 \epsilon_{0} V}\right)[n(n-1) m(m-1)(p+1)]^{1 / 2} e_{\alpha}^{(1)} e_{\beta}^{(1)} e_{\gamma}^{(2)} e_{\delta}^{(2)} \bar{e}_{\epsilon}^{(3)} \bar{e}_{\phi}^{(4)},
$$

where $\boldsymbol{\chi}^{(\xi)}$ is the sixth-rank index-symmetrized six-wave tensor for molecule $\xi$ and $\mathbf{e}^{(n)}$ are the unit polarization vectors for the $n$th mode. In Eq. (2), parentheses amongst indices are used to indicate equivalence of photons. Thus, for example, the parentheses around the $\alpha$ and $\beta$ indices correlate with the invariance of $M_{f i}^{(\xi)}$ with respect to interchange of the vector components $e_{\alpha}^{(1)}$ and $e_{\beta}^{(1)}$. Using perturbation theory the nonsymmetrized six-wave tensor is found to have the form

$$
\chi_{\alpha \beta \gamma \delta \epsilon \phi}^{(\xi)}=\sum_{r, \ldots, \nu}\left\{\frac{\mu_{\phi}^{0 \nu} \mu_{\epsilon}^{\nu u} \mu_{\delta}^{u t} \mu_{\gamma}^{t s} \mu_{\beta}^{s r} \mu_{\alpha}^{r 0}}{\left(\widetilde{E}_{\nu 0}-2 \hbar \omega\right)\left(\widetilde{E}_{u 0}-4 \hbar \omega\right)\left(\widetilde{E}_{t 0}-3 \hbar \omega\right)\left(\widetilde{E}_{s 0}-2 \hbar \omega\right)\left(\widetilde{E}_{r 0}-\hbar \omega\right)}+\cdots\right\},
$$

where we have only shown explicitly the term arising from the Feynman diagram of Fig. 2, the ellipsis representing the 179 terms produced by taking all other distinct time orderings. The energy denominators in Eq. (3) have been written in the complex form $\widetilde{E}_{\nu 0}=E_{\nu}-E_{0}-i \Gamma_{\nu}$ to account for the damping associated with $\Gamma_{\nu}$, the linewidth of the excited state $|\nu\rangle$. Note also that the convention used here for the sign of the damping factor is that determined unequivocally by consideration of time-reversal symmetry. ${ }^{24}$ The symmetrized tensor is now given by the relation

$$
\begin{aligned}
\chi_{(\alpha \beta)(\gamma \delta) \epsilon \phi}^{(\xi)}= & \frac{1}{4}\left(\chi_{\alpha \beta \gamma \delta \epsilon \phi}^{(\xi)}+\chi_{\beta \alpha \gamma \delta \epsilon \phi}^{(\xi)}\right. \\
& \left.+\chi_{\alpha \beta \delta \gamma \epsilon \phi}^{(\xi)}+\chi_{\beta \alpha \delta \gamma \epsilon \phi}^{(\xi)}\right) .
\end{aligned}
$$

The possibility of more extensive index symmetry is an issue we address in Sec. V. At this stage we simply note that an assumption of full Kleinman symmetry, with the extensive simplification that would ensue, could only be warranted in substantially off-resonant applications. ${ }^{25}$

The rate of production of second-harmonic photons into mode 4 is now found by use of the Fermi rule

$$
\Gamma=\frac{2 \pi}{\hbar}\left|\sum_{\xi} M_{f i}^{(\xi)}\right|^{2} \rho_{F},
$$

where $\rho_{F}$ is the density of final (radiation) states. For fluids and other isotropic media this expression must be averaged over the ensemble to arrive at the net response

$$
\langle\Gamma\rangle=\frac{2 \pi \rho_{F}}{\hbar}\left\langle\left|\sum_{\xi} M_{f i}^{(\xi)}\right|^{2}\right\rangle .
$$

The sum over all molecules generates both a diagonal (incoherent) signal derived from individual molecules, and an off- diagonal (coherent) response through the constructive interference of signals from different molecules. For an ensemble consisting of a large number of molecules $N$ the latter strongly dominates and we arrive at a coherent response

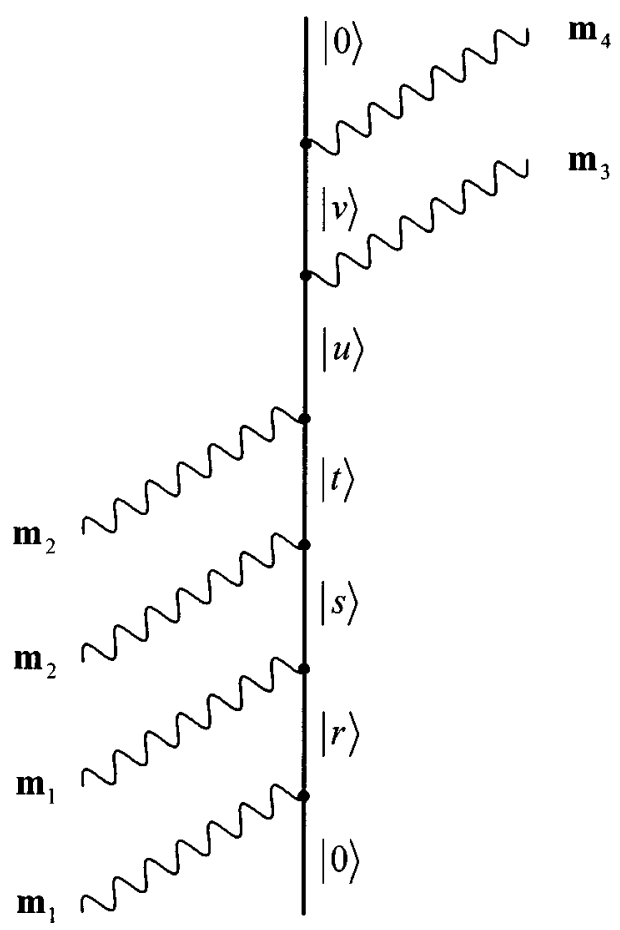

FIG. 2. Representative Feynman diagram labeled according to the modes in which photons are created or annihilated. 


$$
\begin{aligned}
\Gamma_{\mathrm{coh}}= & \frac{2 \pi \rho_{F} N^{2}}{\hbar}\left(\frac{\hbar c k}{2 \epsilon_{0} V}\right)^{4}\left(\frac{\hbar c k^{\prime}}{2 \epsilon_{0} V}\right)^{2} \\
& \times[n(n-1) m(m-1)(p+1)] \\
& \times\left|\left\langle\chi_{(\alpha \beta)(\gamma \delta) \epsilon \phi}\right\rangle e_{\alpha}^{(1)} e_{\beta}^{(1)} e_{\gamma}^{(2)} e_{\delta}^{(2)} \bar{e}_{\epsilon}^{(3)} \bar{e}_{\phi}^{(4)}\right|^{2} .
\end{aligned}
$$

The sixth-rank average is computed using established techniques, ${ }^{26}$ giving

$$
\begin{aligned}
\Gamma_{\mathrm{coh}}= & \frac{2 \pi \rho_{F} N^{2}}{\hbar}\left(\frac{\hbar c k}{2 \epsilon_{0} V}\right)^{4}\left(\frac{\hbar c k^{\prime}}{2 \epsilon_{0} V}\right)^{2} \\
& \times[n(n-1) m(m-1)(p+1)]\left|\sum_{n=1}^{6} \mathrm{X}_{n} E_{n}\right|^{2},
\end{aligned}
$$

where the set of six independent susceptibility invariants is given by the following (using the Einstein convention of implied summation over repeated indices):

$$
\begin{aligned}
& X=\frac{1}{105}\left[\begin{array}{cccccc}
8 & -5 & -5 & 4 & 4 & -5 \\
-5 & 11 & 4 & -6 & -6 & 4 \\
-5 & 4 & 11 & -6 & -6 & 4 \\
4 & -6 & -6 & 16 & 2 & -6 \\
4 & -6 & -6 & 2 & 16 & -6 \\
-5 & 4 & 4 & -6 & -6 & 11
\end{array}\right] \\
& \times\left[\begin{array}{c}
\chi_{(\alpha \alpha)(\beta \beta) \gamma \gamma} \\
\chi_{(\alpha \alpha)(\beta \gamma) \beta \gamma} \\
\chi_{(\alpha \beta)(\alpha \beta) \gamma \gamma} \\
\chi_{(\alpha \beta)(\alpha \gamma) \beta \gamma} \\
\chi_{(\alpha \beta)(\alpha \gamma) \gamma \beta} \\
\chi_{(\alpha \beta)(\gamma \gamma) \alpha \beta}
\end{array}\right],
\end{aligned}
$$

and the corresponding polarization factors are

$$
\left.\begin{array}{ll}
E_{1}=\left(\mathbf{e}_{1} \cdot \mathbf{e}_{1}\right)\left(\mathbf{e}_{2} \cdot \mathbf{e}_{2}\right)\left(\overline{\mathbf{e}}_{3} \cdot \overline{\mathbf{e}}_{4}\right) & E_{2}=\left(\mathbf{e}_{1} \cdot \mathbf{e}_{1}\right)\left(\mathbf{e}_{2} \cdot \overline{\mathbf{e}}_{3}\right)\left(\mathbf{e}_{2} \cdot \overline{\mathbf{e}}_{4}\right) \\
E_{3}=\left(\mathbf{e}_{1} \cdot \mathbf{e}_{2}\right)^{2}\left(\overline{\mathbf{e}}_{3} \cdot \overline{\mathbf{e}}_{4}\right) & E_{4}=\left(\mathbf{e}_{1} \cdot \mathbf{e}_{2}\right)\left(\mathbf{e}_{1} \cdot \overline{\mathbf{e}}_{3}\right)\left(\mathbf{e}_{2} \cdot \overline{\mathbf{e}}_{4}\right) \\
E_{5}=\left(\mathbf{e}_{1} \cdot \mathbf{e}_{2}\right)\left(\mathbf{e}_{1} \cdot \overline{\mathbf{e}}_{4}\right)\left(\mathbf{e}_{2} \cdot \overline{\mathbf{e}}_{3}\right) & E_{6}=\left(\mathbf{e}_{1} \cdot \overline{\mathbf{e}}_{3}\right)\left(\mathbf{e}_{2} \cdot \mathbf{e}_{2}\right)\left(\mathbf{e}_{1} \cdot \overline{\mathbf{e}}_{4}\right)
\end{array}\right\} .
$$

To arrive at an intensity expression we assume that the seeding beam is of sufficiently high intensity that $p \gg 1$, generalize to accommodate radiation fields with arbitrary photon statistics, and we introduce the usual expression for the density of states for the emitted harmonic ${ }^{27}$

$$
\rho_{F}=\frac{\left(k^{\prime}\right)^{2} V d \Omega}{(2 \pi)^{3} \hbar c} .
$$

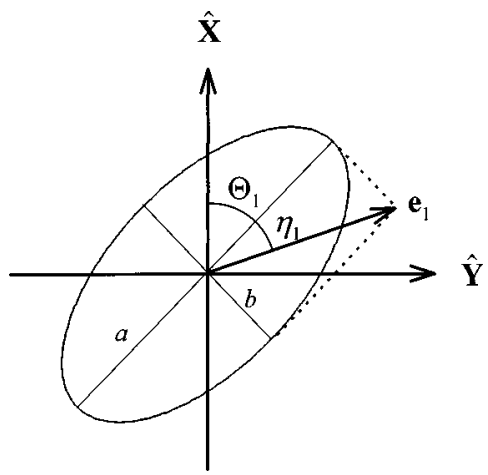

FIG. 3. Definition of the azimuth $\Theta_{1}$ and ellipticity $\eta_{1}=\tan ^{-1}(b / a)$ describing the general polarization state of, in this case, beam 1 . The $\hat{\mathbf{Z}}$ axis here points into the plane of the paper so that beam 1 propagates towards the reader.

The final expression for the signal intensity $I_{\text {sig }}^{(2 \omega)}$ is then expressible as

$$
I_{\mathrm{sig}}^{(2 \omega)}=\frac{\left(k^{\prime}\right)^{4} g_{1}^{(2)} g_{2}^{(2)} N^{2}}{256 \pi^{2} c^{4} \epsilon_{0}^{6}}\left(I_{1}^{(\omega)} I_{2}^{(\omega)}\right)^{2} I_{3}^{(2 \omega)}\left|\sum_{n=1}^{6} \mathrm{X}_{n} E_{n}\right|^{2},
$$

where $g_{i}^{(2)}$ is the degree of second-order coherence of the mode $i$ fundamental beam of mean intensity $I_{i}^{(\omega)}$, and $I_{3}^{(2 \omega)}$ is the intensity of the seeding second harmonic. We are now in a position to derive expressions for the signal intensity for any given arrangement of field polarizations. This we shall do for several configurations that have been studied experimentally: The agreement between theory and experiment proves to be good in all cases.

\section{POLARIZATION ANALYSIS}

Here we are interested only in the polarization characteristics of the second-harmonic signal and so we write Eq. (11) as

$$
I_{\mathrm{sig}}^{(2 \omega)}=\kappa\left|\sum_{n=1}^{6} \mathrm{X}_{n} E_{n}\right|^{2}
$$

The laboratory frame that we shall use is shown in Fig. 1. In this frame the polarization vectors for the four beams, as characterized by their azimuth and ellipticity, defined using the convention shown in Fig. 3, are given by

$$
\left.\begin{array}{l}
\mathbf{e}_{1}=\left(\cos \Theta_{1} \cos \eta_{1}-i \sin \Theta_{1} \sin \eta_{1}\right) \hat{\mathbf{X}}+\left(\sin \Theta_{1} \cos \eta_{1}+i \cos \Theta_{1} \sin \eta_{1}\right) \hat{\mathbf{Y}} \\
\mathbf{e}_{2}=\left(\cos \Theta_{2} \cos \eta_{2}-i \sin \Theta_{2} \sin \eta_{2}\right) \hat{\mathbf{X}}-\left(\sin \Theta_{2} \cos \eta_{2}+i \cos \Theta_{2} \sin \eta_{2}\right) \hat{\mathbf{Y}} \\
\mathbf{e}_{3}=\left(\cos \Theta_{3} \cos \eta_{3}-i \sin \Theta_{3} \sin \eta_{3}\right) \hat{\mathbf{X}}-\left(\sin \Theta_{3} \cos \eta_{3}+i \cos \Theta_{3} \sin \eta_{3}\right)(\cos \delta \hat{\mathbf{Y}}-\sin \delta \hat{\mathbf{Z}}) \\
\mathbf{e}_{4}=\left(\cos \Theta_{4} \cos \eta_{4}-i \sin \Theta_{4} \sin \eta_{4}\right) \hat{\mathbf{X}}+\left(\sin \Theta_{4} \cos \eta_{4}+i \cos \Theta_{4} \sin \eta_{4}\right)(\cos \delta \hat{\mathbf{Y}}-\sin \delta \hat{\mathbf{Z}})
\end{array}\right\} .
$$


The four arrangements used experimentally are shown in Fig. 4. In each case we look at linearly polarized output $\left(\eta_{4}=0\right)$ and measure components of the signal polarized in turn in the $\hat{\mathbf{X}}\left(\Theta_{4}=0\right)$ and $\hat{\mathbf{Y}}\left(\Theta_{4}=\pi / 2\right)$ directions, which we will denote by $I_{\text {sig }}^{X}$ and $I_{\text {sig }}^{Y}$, respectively. There are six combinations of the six-wave tensor that feature in these cases (five of which are linearly independent) and so we define

$$
\left.\begin{array}{ll}
C_{1}=\mathrm{X}_{1}+\mathrm{X}_{2} & C_{4}=2 \mathrm{X}_{1}+\mathrm{X}_{3}+\mathrm{X}_{6} \\
C_{2}=\mathrm{X}_{3}+\mathrm{X}_{4}+\mathrm{X}_{5}+\mathrm{X}_{6} & C_{5}=\mathrm{X}_{3}-\mathrm{X}_{6} \\
C_{3}=\mathrm{X}_{5}+\mathrm{X}_{6} & C_{6}=\mathrm{X}_{4}+\mathrm{X}_{6}=C_{2}-C_{3}-C_{5}
\end{array}\right\} .
$$

In terms of the six tensor combinations given in Eq. (9) we hence find that

$$
\begin{aligned}
{\left[\begin{array}{l}
C_{1} \\
C_{2} \\
C_{3} \\
C_{4} \\
C_{5} \\
C_{6}
\end{array}\right]=} & \frac{1}{105}\left[\begin{array}{cccccc}
3 & 6 & -1 & -2 & -2 & -1 \\
-2 & -4 & 3 & 6 & 6 & 3 \\
-1 & -2 & -2 & -4 & 10 & 5 \\
6 & -2 & 5 & -4 & -4 & 5 \\
0 & 0 & 7 & 0 & 0 & -7 \\
-1 & -2 & -2 & 10 & -4 & 5
\end{array}\right] \\
& \times\left[\begin{array}{c}
\chi_{(\alpha \alpha)(\beta \beta) \gamma \gamma} \\
\chi_{(\alpha \alpha)(\beta \gamma) \beta \gamma} \\
\chi_{(\alpha \beta)(\alpha \beta) \gamma \gamma} \\
\chi_{(\alpha \beta)(\alpha \gamma) \beta \gamma} \\
\chi_{(\alpha \beta)(\alpha \gamma) \gamma \beta} \\
\chi_{(\alpha \beta)(\gamma \gamma) \alpha \beta}
\end{array}\right] .
\end{aligned}
$$

The details of these particular arrangements are as follows:

\section{Case A}

Here, all input beams are linearly polarized along $\hat{\mathbf{X}}$ except beam 1 which makes an angle $\Theta_{1}$ to the others

$$
\left.\begin{array}{l}
I_{\text {sig }}^{X}=\kappa\left|C_{1}+C_{2} \cos ^{2} \Theta_{1}\right|^{2} \\
I_{\text {sig }}^{Y}=\frac{\kappa}{4}\left|C_{3}\right|^{2} \cos ^{2} \delta \sin ^{2} 2 \Theta_{1}
\end{array}\right\} .
$$

\section{Case B}

Here the beams are as in Case A except for beam 2 which is linearly polarized along the $\hat{\mathbf{Y}}$ direction

$$
\left.\begin{array}{l}
I_{\mathrm{sig}}^{X}=\frac{\kappa}{4}\left|C_{4}-C_{5} \cos 2 \Theta_{1}\right|^{2} \\
I_{\mathrm{sig}}^{Y}=\frac{\kappa}{4}\left|C_{6}\right|^{2} \cos ^{2} \delta \sin ^{2} 2 \Theta_{1}
\end{array}\right\} .
$$

\section{Case C}

Input beams are all linearly polarized along $\hat{\mathbf{X}}$ except beam 1 which is changed continuously from linear to circular polarization through the rotation of a $\lambda / 4$ wave plate $\left(\Theta_{1}\right.$ $=\eta_{1}=$ angle of rotation of wave plate from $\hat{\mathbf{X}}$ )

$$
\left.\begin{array}{l}
I_{\mathrm{sig}}^{X}=\frac{\kappa}{4}\left|2\left(C_{1}+C_{2}\right) \cos 2 \Theta_{1}-i C_{2} \sin ^{2} 2 \Theta_{1}\right|^{2} \\
I_{\mathrm{sig}}^{Y}=\frac{\kappa}{4}\left|C_{3}\right|^{2} \cos ^{2} \delta\left(1-\cos ^{4} 2 \Theta_{1}\right)
\end{array}\right\} .
$$

\section{Case D}

Input beams are all linearly polarized along $\hat{\mathbf{X}}$ except beam 1 which is changed continuously from linear to circular polarization through the rotation of a $\lambda / 2$ wave plate $\left(\eta_{1}\right.$ $=$ ellipticity $=$ angle of rotation of wave plate; $\Theta_{1}=0$ )

$$
\left.\begin{array}{l}
I_{\mathrm{sig}}^{X}=\kappa\left|C_{1} \cos 2 \eta_{1}+C_{2} \cos ^{2} \eta_{1}\right|^{2} \\
I_{\mathrm{sig}}^{Y}=\frac{\kappa}{4}\left|C_{3}\right|^{2} \cos ^{2} \delta \sin ^{2} 2 \eta_{1}
\end{array}\right\} .
$$

\section{EXPERIMENT}

The optical arrangement used is shown in Fig. 1. Measurements were made using a regeneratively amplified titanium:sapphire laser producing output pulses at $800 \mathrm{~nm}$ with a repetition frequency of $5 \mathrm{kHz}$ and width $<100 \mathrm{fs}$. The 800 $\mathrm{nm}$ beam was divided into three parts, one of which passed through a BBO second-harmonic generating crystal to yield a beam at $400 \mathrm{~nm}$. The polarization of each beam was independently controlled by broadband half- and quarter-wave plates. Beam 1 was modulated at $500 \mathrm{~Hz}$ by a mechanical chopper and all three beams were temporally and spatially overlapped in the sample with an illuminated area of $\sim 1 \mathrm{~mm}^{2}$. Pulse energies were kept below $60 \mu \mathrm{J}$.

The SWM signal at $400 \mathrm{~nm}$ was detected in the phasematched direction after transmission through the $50 \%$ beam splitter. The signal was then monitored by a photomultiplier tube (PMT) via an analyzing polarizer and a monochromator. The PMT output was measured by a lock-in amplifier referenced to the frequency of the mechanical chopper. Preliminary checks on the intensity of the second-harmonic signal as a function of pump and probe beams confirmed its six-wave origin. In all the experiments reported here the sample was a $100 \mu \mathrm{m}$ pathlength cell containing a $10^{-3} \mathrm{M}$ solution of 4dimethylamino- $4^{\prime}$-nitrostilbene (DMANS) in tetrahydrofuran.

\section{RESULTS AND DISCUSSION}

In our experiments, the sample is absorbing at the second-harmonic frequency $\left(\lambda_{\max }=430 \mathrm{~nm}\right)$. Thus one can expect that some of the sample population will be raised to the excited state during the course of the interaction between the sample and pump pulses. The effect could be explicitly accounted for within the parametric theory presented here by simply modifying Eq. (6) to include a sum over the small subset of excited molecules. However, only if the six-wave tensor associated with these molecules were to be markedly different from that of the ground state would our results need modification. Even in this case the conclusions that follow from Eq. (6) concerning the polarization characteristics of the second-harmonic generation (SHG) signal are unchanged and so we expect the polarization analysis of Sec. III to be valid. 
Equation (3) certainly indicates that in our experiments significant resonance enhancement of the $\boldsymbol{\chi}$ tensor is to be expected. This is simply due to the fact that 96 of the 180 terms contributing to $\boldsymbol{\chi}$ have denominators containing factors of the form $\widetilde{E}_{\nu 0}-2 \hbar \omega=\Delta \omega-i \Gamma_{\nu}$, where $\Delta \omega$ is the detuning. These terms become very large near resonance $(\Delta \omega$ $\rightarrow 0$ ). In this frequency region the tensor $\chi$ can be expected to depart substantially from full index symmetry and its imaginary part will become highly significant. Thus, one would not expect to observe Kleinman symmetry in the experiments we have performed, and the six parameters $\chi_{(\alpha \alpha)(\beta \beta) \gamma \gamma}-\chi_{(\alpha \beta)(\gamma \gamma) \alpha \beta}$ in Eq. (9) should both be complex and differ in magnitude. The second-harmonic intensities collected for Cases A-D are shown in Fig. 5. We first confirm the departure from Kleinman symmetry by noting that if that condition held, the six parameters $\chi_{(\alpha \alpha)(\beta \beta) \gamma \gamma}$ $-\chi_{(\alpha \beta)(\gamma \gamma) \alpha \beta}$ would become equal and, writing $\chi$ $=\chi_{(\alpha \alpha \beta \beta \gamma \gamma)}$, Eq. (15) would then reduce to

$$
\mathbf{C}=\frac{\chi}{35}\left[\begin{array}{llllll}
1 & 4 & 2 & 2 & 0 & 2
\end{array}\right]^{\mathrm{T}} .
$$

An interesting observation here is the disappearance of $C_{5}$. Examination of Eq. (17) indicates that in this case the component of the signal in Case B that is polarized in the $\hat{\mathbf{X}}$ direction should lose its dependence on $\Theta_{1}$. Figure 5 clearly indicates that this is not so, immediately verifying that Kleinman symmetry is not exhibited by our data. We are thus correct to treat the coefficients $C_{1}-C_{6}$ as complex quantities and take this into account when fitting the experimental data to the theoretical expressions given in Eqs. (16)-(19).

The resulting least squares fits are shown in Fig. 5. In each case the number of floating parameters have been kept to a minimum. For example, in Case A, three variables were used to fit the $X$-polarized intensity component and one the Y-polarized component

$$
\begin{aligned}
I_{\text {sig }}^{X}= & N_{X}\left\{\left[1+\operatorname{Re}\left(C_{2} / C_{1}\right) \cos ^{2} \Theta_{1}\right]^{2}\right. \\
& \left.+\left[\operatorname{Im}\left(C_{2} / C_{1}\right) \cos ^{2} \Theta_{1}\right]^{2}\right\}, \\
I_{\text {sig }}^{Y}= & N_{Y} \sin ^{2} 2 \Theta_{1} .
\end{aligned}
$$

The fits are seen to be good in all the cases studied, confirming the theoretical development given here. The $\hat{\mathbf{X}}$-polarized traces are particularly interesting. Equations (16)-(19) indicate that the specific variation of $I_{\text {sig }}^{X}$ in each
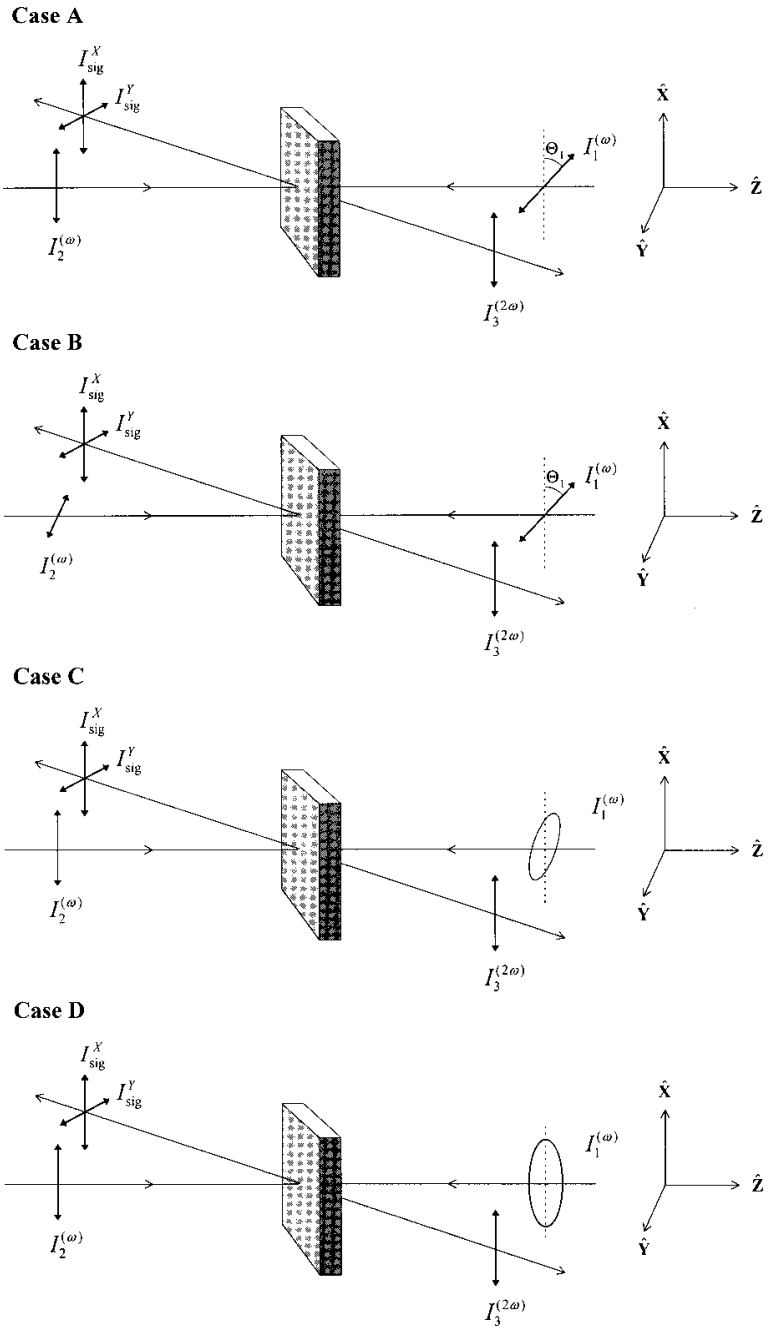

FIG. 4. Four schematic diagrams showing the types of polarization variations used in our experiments. Case A: All beams linearly polarized vertically in the laboratory except beam 1 whose polarization angle is varied; Case B: As for Case A except that the plane of beam 2 is rotated by $\pi / 2$; Case C: Beam 1 changes from vertically linear polarization through elliptical to vertically linear polarization again (the ellipticity is equal to the azimuth throughout); Case D: Beam 1 again goes through elliptical polarization, but now one of the axes of the polarization ellipse is always vertical (azimuth is zero throughout). The beams are identified in each case by their intensity labels.

case depends on one of the ratios $C_{2} / C_{1}$ or $C_{5} / C_{4}$. This permits information to be extracted concerning these ratios. Cases A, C, and D give the overall results

$\operatorname{Re}\left(C_{2} / C_{1}\right)=3.5 \pm 1.5, \quad \operatorname{Im}\left(C_{2} / C_{1}\right)=2.06 \pm 0.63$,

whereas case B yields

$$
\operatorname{Re}\left(C_{5} / C_{4}\right)=-0.111 \pm 0.010, \quad \operatorname{Im}\left(C_{5} / C_{4}\right)= \pm(0.239 \pm 0.071) .
$$

Using Eq. (20) we may recast these results in terms of the six-wave tensor components

$\frac{-2 \chi_{(\alpha \alpha)(\beta \beta) \gamma \gamma}-4 \chi_{(\alpha \alpha)(\beta \gamma) \beta \gamma}+3 \chi_{(\alpha \beta)(\alpha \beta) \gamma \gamma}+6 \chi_{(\alpha \beta)(\alpha \gamma) \beta \gamma}+6 \chi_{(\alpha \beta)(\alpha \gamma) \gamma \beta}+3 \chi_{(\alpha \beta)(\gamma \gamma) \alpha \beta}}{3 \chi_{(\alpha \alpha)(\beta \beta) \gamma \gamma}+6 \chi_{(\alpha \alpha)(\beta \gamma) \beta \gamma}-\chi_{(\alpha \beta)(\alpha \beta) \gamma \gamma}-2 \chi_{(\alpha \beta)(\alpha \gamma) \beta \gamma}-2 \chi_{(\alpha \beta)(\alpha \gamma) \gamma \beta}-\chi_{(\alpha \beta)(\gamma \gamma) \alpha \beta}}=(3.5 \pm 1.5)+i(2.06 \pm 0.63)$, 

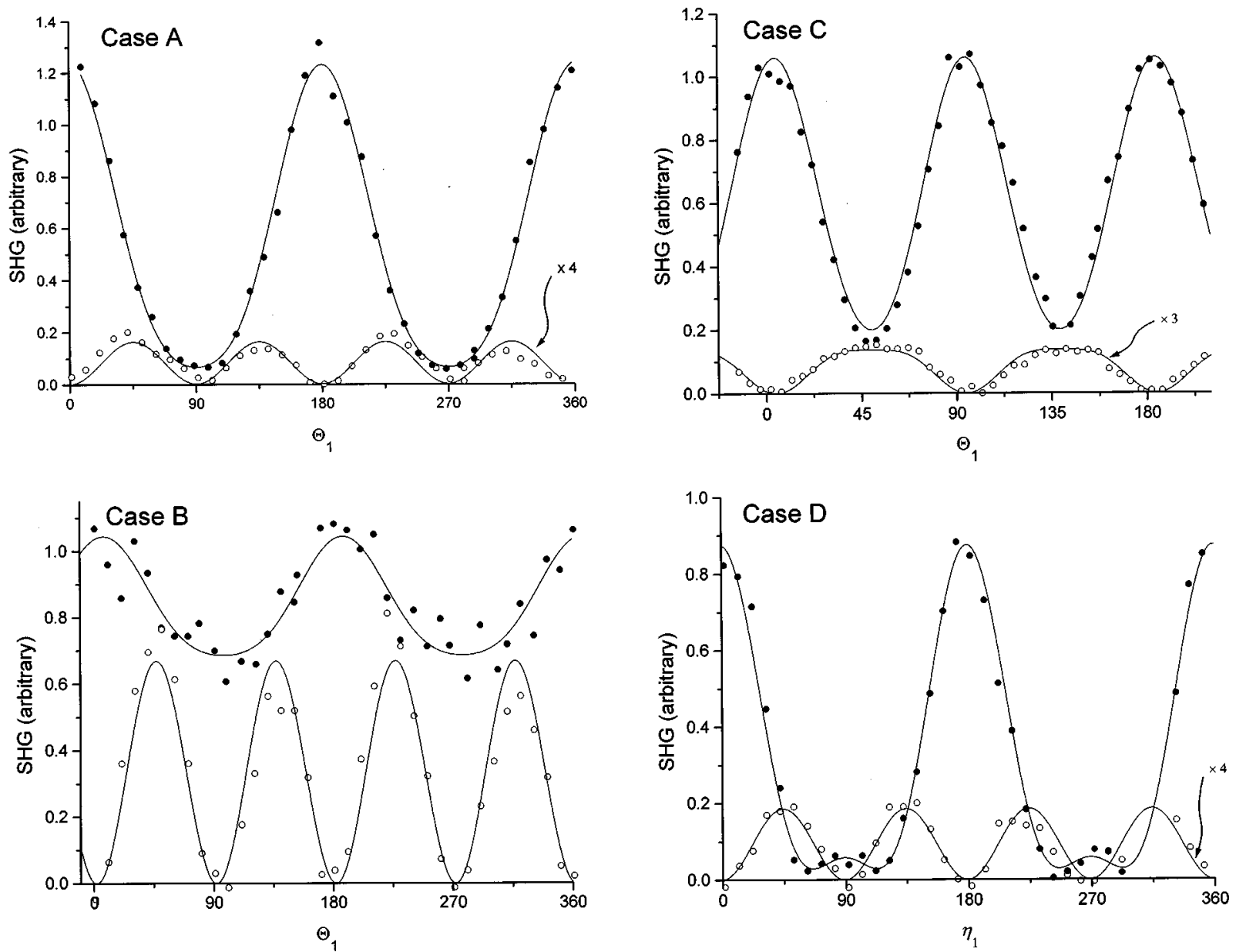

FIG. 5. Experimental results and least squares fits to the theoretical expressions, Eqs. (16)-(19). The cases correspond to those shown in Fig. 4 -filled circles denoting the vertically polarized signal $I_{\text {sig }}^{X}$ and open circles the horizontally polarized signal $I_{\text {sig }}^{Y}$.

$\frac{7 \chi_{(\alpha \beta)(\alpha \beta) \gamma \gamma}-7 \chi_{(\alpha \beta)(\gamma \gamma) \alpha \beta}}{6 \chi_{(\alpha \alpha)(\beta \beta) \gamma \gamma}-2 \chi_{(\alpha \alpha)(\beta \gamma) \beta \gamma}+5 \chi_{(\alpha \beta)(\alpha \beta) \gamma \gamma}-4 \chi_{(\alpha \beta)(\alpha \gamma) \beta \gamma}-4 \chi_{(\alpha \beta)(\alpha \gamma) \gamma \beta}+5 \chi_{(\alpha \beta)(\gamma \gamma) \alpha \beta}}$
$=(-0.111 \pm 0.010) \pm i(0.239 \pm 0.071)$.

The nonzero imaginary components here strongly signal, once again, the departure from Kleinman symmetry. The values derived here can of course be supplemented by similar ratios for other components of $\boldsymbol{\chi}$ by adopting different experimental arrangements. Six-wave mixing experiments, such as those we describe, are thus capable of producing valuable and highly detailed information about the six-wave tensor. In this respect they may also serve as critical tests of accuracy of ab initio or semiempirical computations.

The given theory has as its premise the simultaneous availability of photons in modes $\mathbf{m}_{1}, \mathbf{m}_{2}$, and $\mathbf{m}_{3}$. This condition is, of course, satisfied when all of the generating pulses arrive at the sample synchronously, as in the experiments discussed. However, by introducing time delays between the pulses we can examine the situation wherein this condition is violated. Experiments relating to a delay of beam 1 (in the notation of Fig. 1) have recently been reported. ${ }^{4,11}$ The analysis of these experiments requires a modification of the theory outlined above. For example, the time-resolved SHG data can be understood as arising from the formation of an anisotropic spatial grating in the medium by beams 2 and 3 , and its subsequent decay, as monitored by the time-delayed beam 1. A quantum electrodynamical treatment of this transient grating scattering mechanism forms the subject of a separate paper. ${ }^{18}$

In summary, we have described a phase-conjugated sixwave mixing experiment and presented data for the signal intensity as a function of beam polarization for a variety of configurations. Using the theory of quantum electrodynamics we have derived expressions for the signal intensity and shown that the theory gives a good account of the experimental data. Furthermore we have used the theory to derive highly intricate information on the high order six-wave tensor responsible for the process in DMANS. 


\section{ACKNOWLEDGMENT}

We are happy to acknowledge the Engineering and Physical Sciences Research Council of the United Kingdom for provision of funding for this and ongoing work.

${ }^{1}$ Y. Tanimura and S. Mukamel, J. Chem. Phys. 99, 9496 (1993).

${ }^{2}$ S. Mukamel, Principles of Nonlinear Optics (OUP, New York, 1995).

${ }^{3}$ Ultrafast Phenomena X (Springer, Berlin, 1996).

${ }^{4}$ S. Lin, D. L. Andrews, I. D. Hands, and S. R. Meech, Chem. Phys. Lett. 285, 321 (1998).

${ }^{5}$ K. Tominaga and K. Yoshihara, Phys. Rev. Lett. 74, 3061 (1995).

${ }^{6} \mathrm{~K}$. Tominaga and K. Yoshihara, J. Chem. Phys. 104, 1159 (1996).

${ }^{7}$ K. Tominaga and K. Yoshihara, J. Chem. Phys. 104, 4419 (1996).

${ }^{8}$ T. Steffen and K. Duppen, Phys. Rev. Lett. 76, 1224 (1996).

${ }^{9}$ T. Steffen and K. Duppen, J. Chem. Phys. 106, 3854 (1997).

${ }^{10}$ S. Lin, D. L. Andrews, I. D. Hands, and S. R. Meech, Chem. Phys. Lett. 285, 321 (1998).

${ }^{11}$ C. Fiorini, F. Charra, and J. M. Nunzi, J. Opt. Soc. Am. B 11, 2347 (1994).

${ }^{12}$ U. Österberg and W. Margulis, Opt. Lett. 7, 310 (1982).

${ }^{13}$ N. B. Baranova and B. Ya. Zel'dovich, JETP Lett. 45, 717 (1987).
${ }^{14}$ F. Charra, F. Devaux, J. M. Nunzi, and P. Raimond, Phys. Rev. Lett. 68, 2440 (1992).

${ }^{15}$ F. Charra, F. Kajzar, J. M. Nunzi, P. Raimond, and E. Idiart, Opt. Lett. 18, 941 (1993).

${ }^{16}$ D. L. Andrews, Nonlinear Opt. 8, 25 (1994).

${ }^{17}$ C. Fiorini, F. Charra, J. M. Nunzi, and P. Raimond, J. Opt. Soc. Am. B 14, 1984 (1997).

${ }^{18}$ I. D. Hands, S. Lin, S. R. Meech, and D. L. Andrews (work in progress).

${ }^{19}$ T. Steffen, J. T. Fourkas, and K. Duppen, J. Chem. Phys. 105, 7364 (1996).

${ }^{20}$ A. Tokmakoff, J. Chem. Phys. 105, 13 (1996).

${ }^{21}$ V. Khidekel, V. Chernyak, and S. Mukamel, J. Chem. Phys. 105, 8543 (1996).

${ }^{22}$ R. L. Murray and J. T. Fourkas, J. Chem. Phys. 107, 9726 (1997).

${ }^{23}$ M. Pfeiffer and A. Lau, J. Chem. Phys. 108, 4159 (1998).

${ }^{24}$ D. L. Andrews, S. Naguleswaran, and G. E. Stedman, Phys. Rev. A 57, 4925 (1998).

${ }^{25}$ G. Wagniére, Appl. Phys. B: Photophys. Laser Chem. 41, 169 (1986).

${ }^{26}$ D. L. Andrews and T. Thirunamachandran, J. Chem. Phys. 67, 5026 (1977).

${ }^{27}$ E. A. Power, Introductory Quantum Electrodynamics (Longmans, London, 1964), p. 93. 